\title{
A novel CD34-specific T-cell engager efficiently depletes acute myeloid leukemia and leukemic stem cells in vitro and in vivo
}

Lucas C. M. Arruda, ${ }^{1}$ Arwen Stikvoort, ${ }^{1}$ Melanie Lambert, ${ }^{2}$ Liqing Jin, ${ }^{3}$ Laura Sanchez Rivera, ${ }^{4}$ Renato M. P. Alves, ${ }^{4}$ Tales Rocha de Moura, ${ }^{5}$ Carsten Mim, ${ }^{5}$ Sören Lehmann, ${ }^{2}$ Rebecca Axelsson-Robertson, ${ }^{1,6}$ John E. Dick, ${ }^{3,7}$ Jonas Mattsson, ${ }^{3,8}$ Björn Önfelt, ${ }^{4,9}$ Mattias Carlsten ${ }^{2,10}$ and Michael Uhlin $1,4,6$

${ }^{1}$ Department of Clinical Science, Intervention and Technology, Karolinska Institute, Stockholm, Sweden; ${ }^{2}$ Center for Hematology and Regenerative Medicine, Department of Medicine, Huddinge, Karolinska Institutet, Stockholm, Sweden; ${ }^{3}$ Princess Margaret Cancer Centre, University Health Network, Toronto, Ontario, Canada; ${ }^{4}$ Department of Applied Physics, Science for Life Laboratory, KTH Royal Institute of Technology, Stockholm, Sweden; ${ }^{5}$ Department for Biomedical Engineering and Health Systems, KTH Royal Institute of Technology, Stockholm, Sweden; ${ }^{6}$ Department of Immunology and Transfusion Medicine, Karolinska University Hospital, Stockholm, Sweden; ${ }^{7}$ Department of Molecular Genetics, University of Toronto, Ontario, Canada; ${ }^{8}$ Gloria and Seymour Epstein Chair in Cell Therapy and Transplantation, Princess Margaret Cancer Centre, University Health Network, Toronto, Ontario, Canada; ${ }^{9}$ Department of Microbiology, Tumor and Cell Biology, Science for Life Laboratory, Karolinska Institutet, Stockholm, Sweden and ${ }^{10} \mathrm{Center}$ for Cell Therapy and Allogeneic Stem Cell Transplantation, Karolinska University Hospital, Stockholm, Sweden.

\section{Correspondence:}

Lucas C. M. Arruda lucas.arruda@ki.se

$\begin{array}{ll}\text { Received: } & \text { June 23, } 2021 . \\ \text { Accepted: } & \text { February } 1,2022 . \\ \text { Prepublished: } & \text { February 10, } 2022 . \\ \text { https://doi.org/10.3324/haematol.2021.279486 } \\ \text { (C2022 Ferrata Storti Foundation } \\ \text { Haematologica material is published under } \\ \text { a CC-BY-NC license @) (1) } \$\end{array}$

\begin{abstract}
Less than a third of patients with acute myeloid leukemia (AML) are cured by chemotherapy and/or hematopoietic stem cell transplantation, highlighting the need to develop more efficient drugs. The low efficacy of standard treatments is associated with inadequate depletion of $\mathrm{CD} 34^{+}$blasts and leukemic stem cells, the latter a drug-resistant subpopulation of leukemia cells characterized by the $\mathrm{CD} 34^{+} \mathrm{CD} 38^{-}$phenotype. To target these drug-resistant primitive leukemic cells better, we have designed a CD34/CD3 bi-specific T-cell engager (BTE) and characterized its anti-leukemia potential in vitro, ex vivo and in vivo. Our results show that this CD34-specific BTE induces CD34-dependent T-cell activation and subsequent leukemia cell killing in a dose-dependent manner, further corroborated by enhanced T-cell-mediated killing at the singlecell level. Additionally, the BTE triggered efficient T-cell-mediated depletion of CD34+ hematopoietic stem cells from peripheral blood stem cell grafts and $\mathrm{CD} 34^{+}$blasts from AML patients. Using a humanized AML xenograft model, we confirmed that the CD34-specific BTE had in vivo efficacy by depleting CD34+ blasts and leukemic stem cells without side effects. Taken together, these data demonstrate that the CD34-specific BTE has robust antitumor effects, supporting development of a novel treatment modality with the aim of improving outcomes of patients with AML and myelodysplastic syndromes.
\end{abstract}

\section{Introduction}

Acute myeloid leukemia (AML) and high-risk myelodysplastic syndromes (MDS) are poor prognosis hematologic malignancies characterized by abnormal hematopoiesis and dysfunctions of the hematopoietic stem cell (HSC) system. ${ }^{1,2}$ Chemotherapy remains the standard of care but is associated with side effects and often high rates of relapse. At present, less than a third of patients diagnosed with AML are cured. ${ }^{3}$ Resistance to standard treatment has recently been attributed to inadequate depletion of leukemic stem cells (LSC), a self-renewing population of leukemic cell progenitors characterized, typically but not exclusively, by the CD34 ${ }^{+}$DD $38^{-}$phenotype. ${ }^{4-6}$ Relapsefated LSC have been identified in combined stem cell/clonal evolution studies of paired diagnosis-relapse samples ${ }^{7}$ and the survival of phenotypic LSC after chemotherapy is associated with minimal residual disease and subsequent relapse. ${ }^{8}$ LSC-specific gene expression signatures $^{9}$ and high LSC burden are associated with high risk of relapse and death..$^{10-13}$ Hence, LSC represent optimal targets for new therapies aimed at improving the outcome of patients with AML/MDS. ${ }^{14}$

$\mathrm{Bi}$-specific T-cell engagers (BTE) are promising immuno- 
therapeutic agents intended for cancer treatment. BTE are small molecules constructed of two single chain variable fragments (scFv) connected in tandem by a flexible linker that acts by retargeting $T$ cells against tumor cells..$^{15}$ One scFv binds to CD3, while the second scFv binds to a tumor-associated antigen. ${ }^{16}$ This structure and specificity allow a BTE construct to physically link a T cell to a tumor cell, stimulating effector cell activation and ultimately leading to cytokine production and tumor killing. ${ }^{17}$ The arrangement of antigen-binding sites and the flexible linker forces target and effector cells into very close proximity, leading to the formation of a cytolytic immune synapse that can counteract the multiple mechanisms of immune evasion presented by cancer cells, such as MHC downregulation. ${ }^{15,16}$ Several studies have shown that LSC are resistant to conventional chemotherapy and radiation-based therapies and to NK-/T-cell-mediated cytotoxicity ${ }^{18-20}$ due to drug extrusion ${ }^{20}$ and low immunogenicity, resulting from low expression of major immune response molecules. ${ }^{18} \mathrm{~A}$ LSC-targeting BTE could efficiently bypass this and mediate immune activation towards LSC, thereby being a more specific and less toxic treatment than those currently available. ${ }^{15}$ The CD19/CD3 BTE blinatumomab has been used to treat B-cell acute lymphoblastic leukemia with great success, ${ }^{21-23}$ representing a great advance on the use of BTE to treat blood cancers.

Leukemic cells express several antigens that are under investigation for targeted immunotherapies, such as CD33. Gemtuzumab (an anti-CD33 monoclonal antibody) was recently approved by the Food and Drug Administration for the treatment of relapsed/refractory $\mathrm{CD} 33^{+} \mathrm{AML},{ }^{24}$ being a very significant step toward defining new immunotherapeutic treatment regimens in AML. Several BTE have been investigated to treat $A M L$ and MDS, targeting $C D 33,{ }^{25}$ CD123 ${ }^{26}$ and WT1, ${ }^{27}$ but no CD34-specific BTE has been developed. CD34 is a molecule expressed almost exclusively by normal stem and progenitor cells but is also found in AML/MDS blasts, and to a lesser degree on renal vessel walls. CD34 is highly expressed by AML blasts and is associated with several genetic aberrancies characteristic of the development of AML. ${ }^{28}$ CD34 expression has been linked to increased resistance to apoptosis and to multidrug resistance, ${ }^{29}$ protecting $C D 34^{+}$blasts from the immune system and chemotherapeutic drugs. Because of such characteristics, CD34 expression is associated with low survival and high relapse rates. ${ }^{11-13,28}$ Indeed, the presence of $\mathrm{CD}_{34}{ }^{+}$leukemic cells was associated with an approximately 4-fold lower event-free survival than in patients with absence of $\mathrm{CD} 34^{+}$leukemic cells, ${ }^{28}$ indicating that this molecule is a promising target to improve AML treatment.

Here, we demonstrate the preclinical efficacy of a CD34specific BTE to target CD34+ AML cells in vitro and in vivo. We observed that the BTE promoted specific killing of
AML cell lines and AML blasts by T-cell-dependent mechanisms. The antibody showed no toxicity in AML-bearing immune-deficient mice infused with human T cells, making this bi-specific antibody a very attractive clinical candidate with potential to improve the outcome of patients with AML and MDS.

\section{Methods}

\section{T-cell isolation and in vitro killing assay}

T-cell cytotoxicity was assessed by fluorescence activated cell sorting (FACS) using purified $\mathrm{T}$ cells from peripheral blood mononuclear cells as effector cells and cancer cell lines (KG1a, Kasumi 1, NALM-6), primary AML blasts or hCMEC/D3 as target cells. Cells were co-cultured at an effector-to-target ratio (E.T) of $3: 1$ for $48 \mathrm{~h}$ (cancer cell lines and hCMEC/D3) and $72 \mathrm{~h}$ (primary AML samples), and serial dilutions of $\alpha$ CD34, CD34/CD3 and RSV/CD3 BTE. Target cell killing was assessed by gating on the CellTrace ${ }^{+}$ fraction and evaluating the annexin $V$ and 7-aminoactinomysin D staining. ${ }^{30,31}$ For more information see the Online Supplementary Appendix.

\section{Proliferation assay}

Purified T cells were stained with $2 \mu \mathrm{M}$ of CellTrace violet (ThermoFisher) according to the manufacturer's instructions and incubated with target cells (KG1a, Kasumi 1, NALM-6) and serial dilutions of $\alpha$ CD34, CD34/CD3 and RSV/CD3 BTE for 5 days at $37^{\circ} \mathrm{C}$ in complete RPMI medium. The fraction of CellTrace ${ }^{\text {low }}$ proliferating $\mathrm{T}$ cells was assessed by FACS.

\section{Single-cell killing assay}

Time-lapse live single-cell imaging was performed as previously described. ${ }^{32,33}$ A detailed description is provided in the Online Supplementary Appendix.

\section{Human primary acute myeloid leukemia samples}

The blood samples from AML patients were collected at Princess Margaret Cancer Center (Toronto, Canada) and CD34+ AML blasts were used as target cells in the killing assays. Purified T cells from donor lymphocyte infusions were used as effector cells. Bone marrow samples from three AML patients were collected at Karolinska University Hospital (Huddinge, Sweden). Cells were co-cultured with T cells $(n=5)$ isolated from heathy donor buffy coats in an E:T of $3: 1$ for $72 \mathrm{~h}$ in the presence of serial dilutions of CD34/CD3 BTE. All patients provided informed consent in accordance with the Declaration of Helsinki and with approval of the ethics committees in the respective centers (ethical approval 2010-1496-31-3). After incubation, AML blast cell depletion and T-cell activation were assessed by FACS. See the Online Supplementary Appendix for details. 


\section{Human hematopoietic stem cell depletion assay}

Unmanipulated peripheral blood stem cell samples were collected during preparation of grafts for hematopoietic stem cell transplantation (HSCT) and processed as previously described. ${ }^{34}$ HSC were purified by positive selection of $\mathrm{CD} 34^{+}$cells using the CD34 MicroBead Kit UltraPure (Miltenyi Biotec). T cells were purified from the same graft using negative selection (Pan T-Cell Isolation Kit; Miltenyi Biotec). Immediately after purification, $T$ cells and HSC were seeded at a 3:1 ratio and cultured for $48 \mathrm{~h}$ with serial dilutions of $\alpha C D 34$, CD34/CD3 and RSV/CD3 BTE. T-cell activation was assessed by CD25 and CD69 expression and depletion of $\mathrm{CD} 34^{+} \mathrm{HSC}$ by gating the CD $45^{\mathrm{dim}} \mathrm{CD} 34^{+}$population. See the Online Supplementary Appendix for details.

\section{In vivo xenograft model}

The antitumor efficacy and safety of a CD34-specific BTE were evaluated in 6- to 8-week-old NOD.CgPrkdc scidil2rg ${ }^{\text {tmiwjl }} \mathrm{Tg}$ (CMV-IL3,CSF2,KITLG)1Eav/MloySzJ (NSG-SGM3) mice (Taconic). All the experiments were performed according to the Swedish Animal Welfare Ordinance and approved by the local animal ethics committee (ethical approval ID1533). See the Online Supplementary Appendix for details.

\section{Statistical analysis}

In vitro data were analyzed using the Wilcoxon matched pairs signed rank-test. In vivo data from mice were compared using an unpaired Student $t$-test. Correlations were assessed using a non-parametric Spearman rank correlation coefficient. GraphPad Prism 9.0 was used, with significance set at $P \leq 0.05$.

\section{Results}

\section{Design and expression of CD34/CD3 bi-specific T-cell} engagers and controls

CD34/CD3 and RSV/CD3 BTE were assembled from two scFv domains by recombinant DNA technology (Online Supplementary Figure S1A, B). The SCFV domain binding to CD34 is positioned $\mathrm{N}$-terminally, and the SCFv binding to CD3 $\mathrm{C}$-terminally, followed by a hexa-histidine sequence. FACS analysis demonstrated that the CD34/CD3 BTE is capable of binding to the CD34+ AML cell lines KG1a and Kasumi 1 as well as to purified human T cells (Online Supplementary Figure S1C, D). We found no cross-reactivity against mouse CD34 (Online Supplementary Figure S1E). The RSV/CD3 control BTE have exactly the same CD $3 \varepsilon$ SCFv binding domain as that of the CD34/CD3 BTE in addition to the RSV-specific ScFV domain, allowing us to assess whether target specificity is required for the T-cell activation. The $\alpha C D 34$ unit, which binds to the target protein but does not trigger CD3 activation, was also developed as a control.

\section{The CD34-specific bi-specific T-cell engager redirects $\mathrm{T}$-cell effector function towards acute myeloid leukemia cell lines}

We next addressed whether the CD34/CD3 BTE was able to redirect primary human $T$ cells to deplete $C D 34^{+}$ human AML cell lines. Initially, we assessed the T-cell activation and killing dynamics by co-culturing effector $T$ cells and target AML/acute lymphoblastic leukemia cell lines at an E:T ratio of 3:1 for a time range between $24 \mathrm{~h}$ and $120 \mathrm{~h}$ (Online Supplementary Figure S2). We observed that $48 \mathrm{~h}$ was the optimal timing to assess Tcell effector function and the earliest timepoint at which maximum killing is observed, being adopted thereafter. By co-culturing $T$ cells and target cell lines for $48 \mathrm{~h}$ in the presence of increasing concentrations of BTE or controls, we observed that CD34-BTE efficiently triggered T-cell-mediated depletion of the CD34 $4^{\text {hi }} \mathrm{KG1a}$ and CD34 ${ }^{\text {low }}$ Kasumi 1 cell lines, while both RSV/CD3 and $\alpha C D 34$ controls killed none of the target cell lines (Figure 1A, B). The CD34/CD3 BTE did not trigger any unspecific killing of CD34- cells as shown using the CD34- human cell line NALM-6 (Figure 1C), indicating that CD34/CD3 BTE killing is specific to CD34+ cells.

\section{The CD34 bi-specific T-cell engager mediates cell- cell interactions and target cell killing}

In order to understand better how the CD34 BTE affects T-cell dynamics we used a single-cell microchip-based method for live cell imaging (Online Supplementary Figure S3). ${ }^{32,33}$ Effector $\mathrm{T}$ cells and target KG1a cells were stained with distinct dyes and seeded into a siliconglass microchip containing thousands of wells, in which interactions between effector and target cells can be followed by live imaging (Online Supplementary Figure S3). T-cell interaction dynamics and target cell lysis were assessed without or in the presence of CD34/CD3 or RSV/CD3. The presence of the CD34/CD3 BTE led to significant increases in cytotoxicity (Figure $2 \mathrm{~A}$ ) and fraction of lytic contacts (Figure 2B) as compared to RSV/CD3 or untreated conditions. The CD34/CD3 BTE also resulted in longer lytic and non-lytic contacts between $T$ cells and target cells as compared to the other conditions (Figure 2C). The BTE also resulted in fewer contacts being formed (Figure 2D), which is a sign of the BTE mediating more stable contacts, and a shorter time until the $T$ cell makes the first contact with target cells (Figure 2E). Taken together, our data show that the BTE led to formation of stable contacts between T cells and target cells often resulting in target cell lysis. 

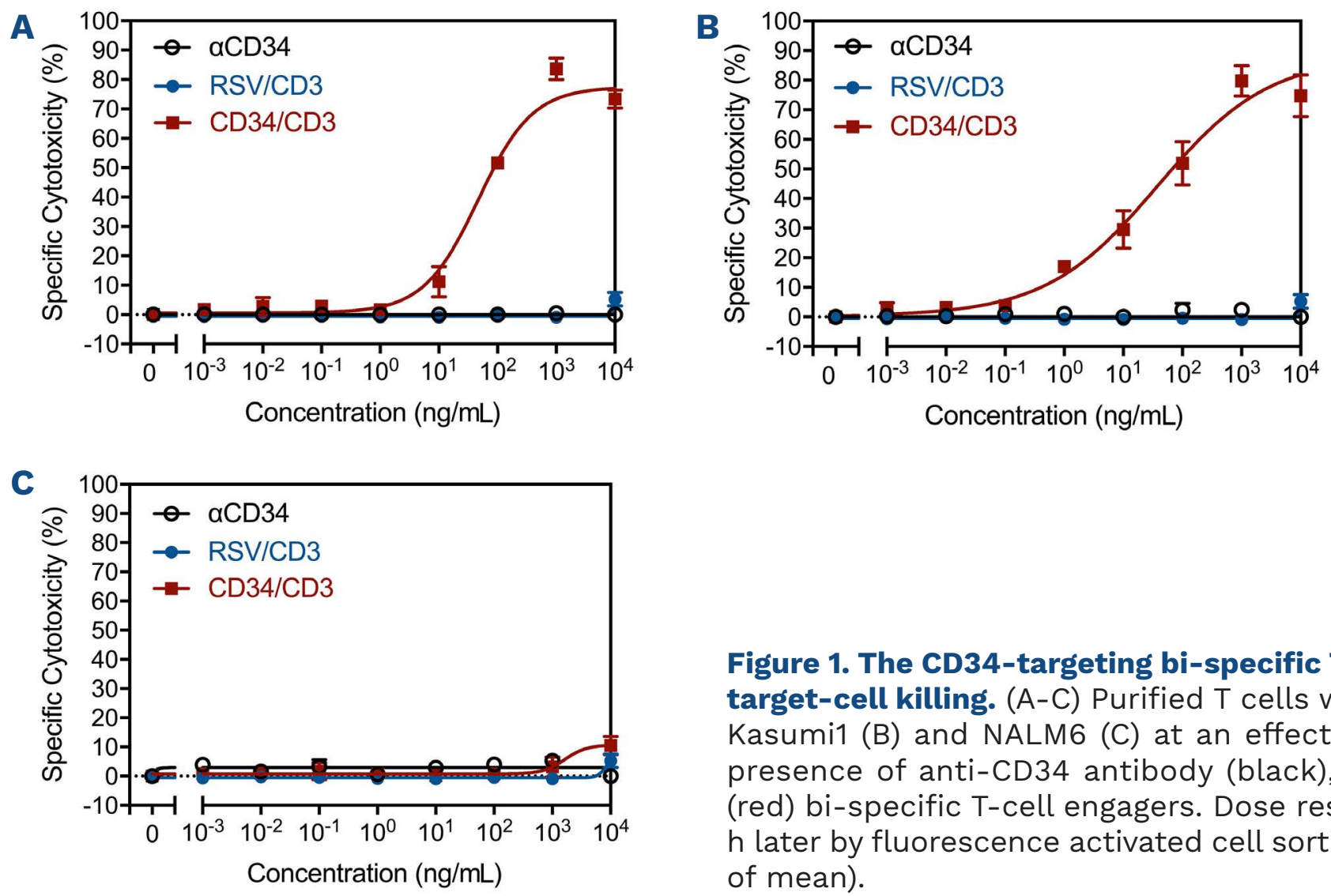

Figure 1. The CD34-targeting bi-specific T-cell engager mediates CD34+ target-cell killing. (A-C) Purified T cells were co-cultured with KG1a (A), Kasumi1 (B) and NALM6 (C) at an effector-to-target ratio of 3:1 in the presence of anti-CD34 antibody (black), RSV/CD3 (blue) or CD34/CD3 (red) bi-specific T-cell engagers. Dose response killing was assessed 48 $h$ later by fluorescence activated cell sorting $(n=3$, mean \pm standard error of mean).
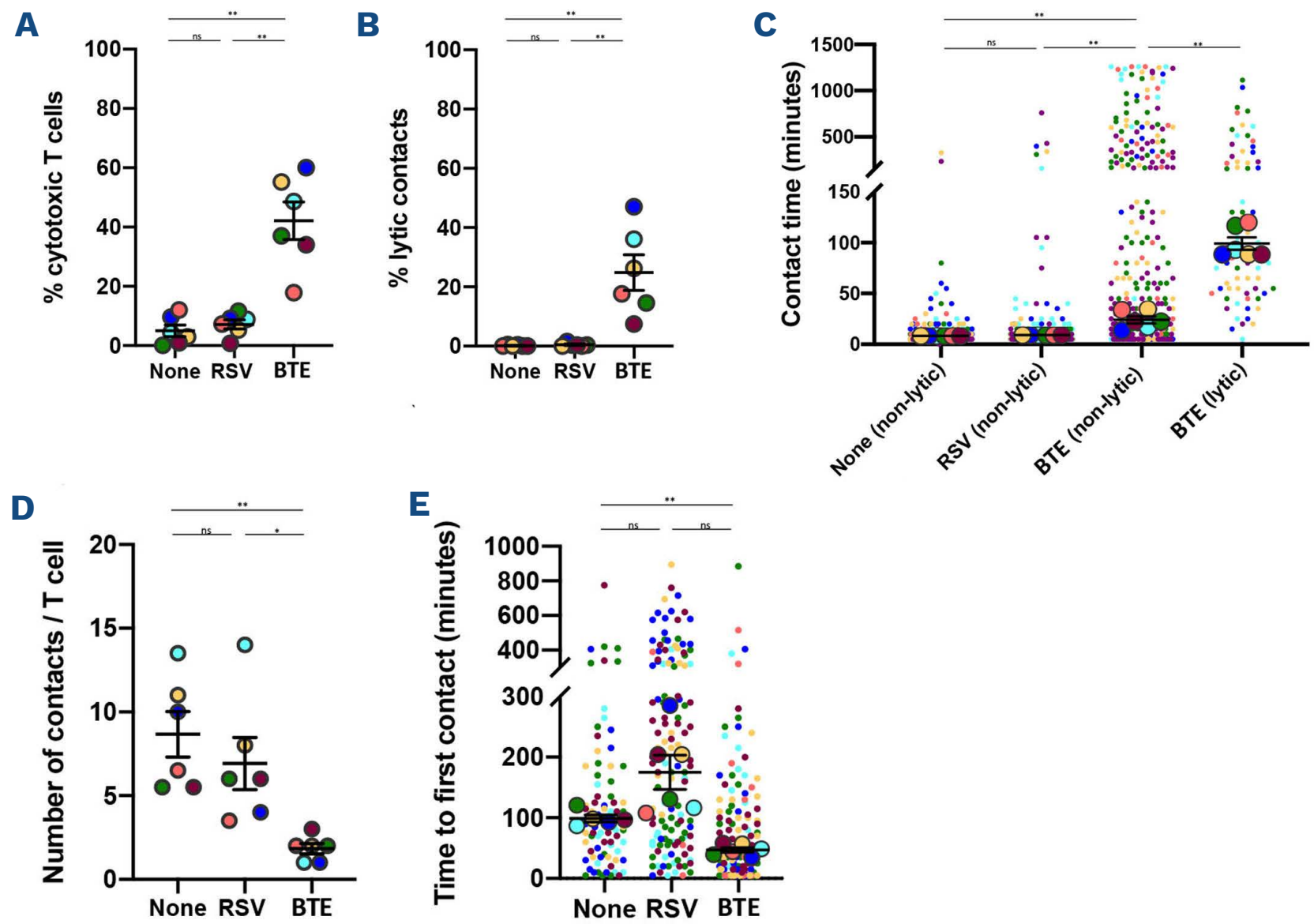

Figure 2. CD34-specific bi-specific T-cell engager mediates stronger cell-cell contact and T-cell killing. Primary $T$ cells were purified and seeded with KG1a target cells onto a silicon-glass microchip containing CD34-specific (bi-specific T-cell engager, BTE) or RSV-specific antibodies (RSV) or left untreated (none). Images were acquired every 5 minutes over 21 hours. (A) Percentage of cytotoxic T cells ( $n=6$; mean \pm standard error of mean [SEM]). (B) Percentage of all contacts that led to target cell death ( $n=6$; mean \pm SEM). (C) Duration of the contacts for the different treatments (none, RSV, BTE). For the BTE condition contacts have been divided into non-lytic (no target cell death) and lytic (target cell death). Individual dots of the same color represent single contact events from one donor, and the bigger circles of the same color represent the median time for that donor ( $n=6$; mean \pm SEM), differences between conditions were evaluated by a paired $t$-test using the median values. (D) Number of contacts per $T$ cell (each circle depicts the median number of events per donor, $n=6$; mean \pm SEM). (E) Time from the beginning of the experiment until the $T$ cell makes the first contact with the target cell (individual dots of the same color represent the first contact in a specific well from one donor, and the bigger circles of the same color represent the median time for that donor ( $n=6$; mean \pm SEM), differences between conditions were evaluated by a paired $t$-test using the median values). Each color represents a different donor $(n=6)$, and color coding is maintained in all figures. Statistical significance: ${ }^{\star} P<0.05$. $* \star P<0.01$. ns: non-significant (paired t-test). 
A

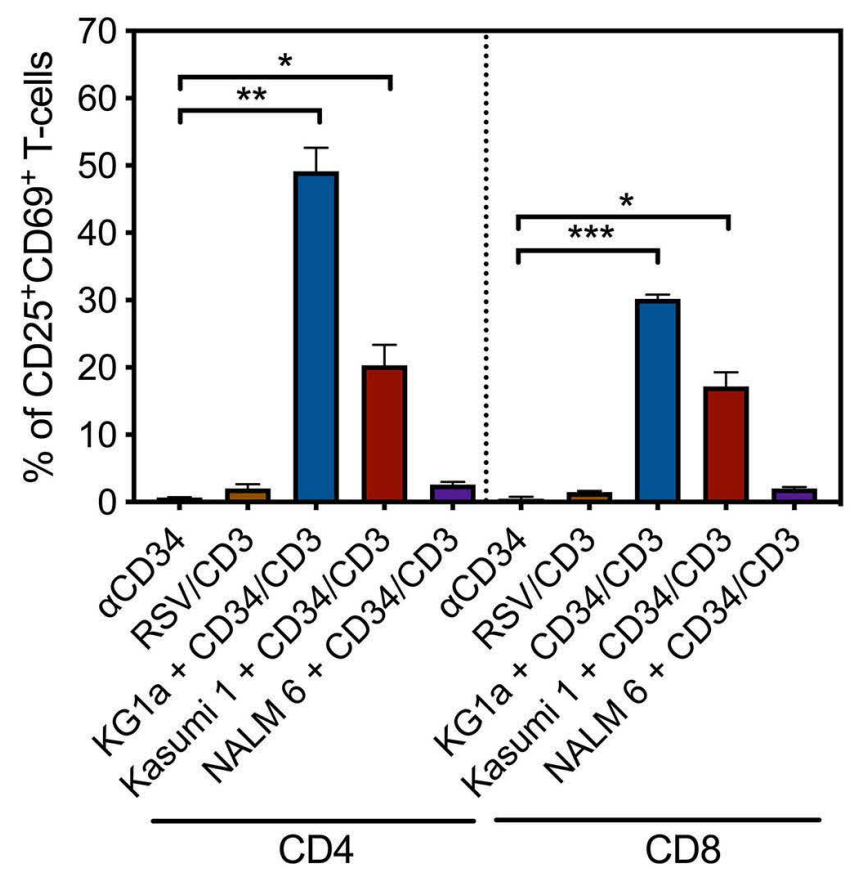

B

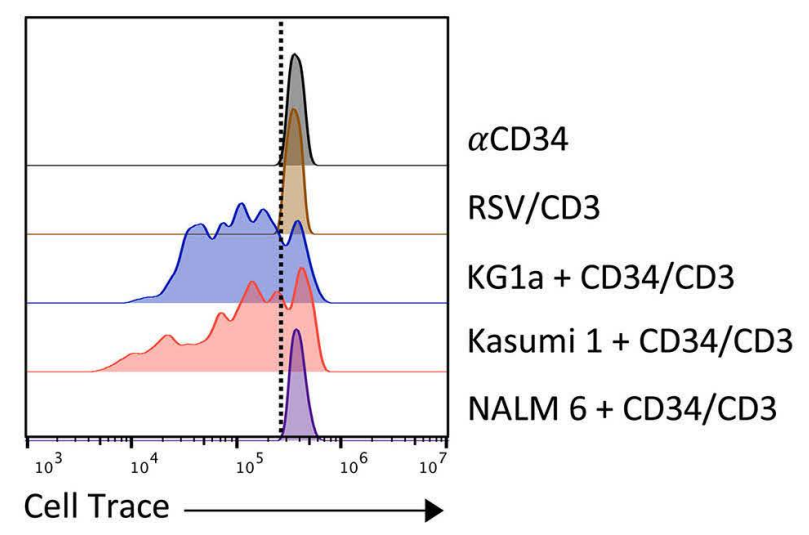

C

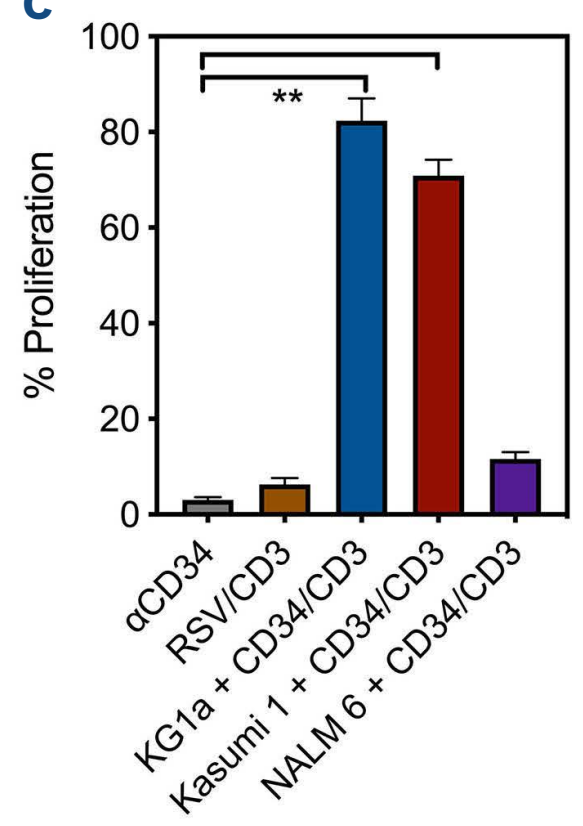

Figure 3. Bi-specific T-cell engager-induced interaction and killing leads to T-cell activation and proliferation. Purified $T$ cells were co-cultured with KG1a, Kasumi1 and NALM6 in the presence of anti-CD34 antibody, RSV/CD3 or CD34/CD3 bi-specific Tcell engager (BTE) at the concentration of $100 \mathrm{ng} / \mathrm{mL}$. (A) $\mathrm{CD} 3^{+} \mathrm{CD} 4^{+}\left(\mathrm{CD} 8^{+}\right) \mathrm{CD} 25^{+} \mathrm{CD} 69^{+}$activated $\mathrm{T}$ cells were assessed after $48 \mathrm{~h}$ of co-culture (mean \pm standard error of mean [SEM], $n=3$ ). (B) Representative histogram overlays of proliferation-induced CellTrace dilution on $\mathrm{CD}^{+} \mathrm{T}$ cells after 5 days of co-culture. (C) Proliferation quantification gated as CellTrace ${ }^{\text {low }} \mathrm{T}$ cells (mean $\pm \mathrm{SEM}$, $\mathrm{n}=3$ ). $\star P<0.05, * \star P<0.01, * \star \star P<0.001$ (Wilcoxon test).

\section{Bi-specific T-cell engager-mediated T-cell activation and proliferation are CD34-dependent}

Next, we examined T-cell activation and proliferation. We observed that both $C D 4^{+}$and $C D 8^{+} \mathrm{T}$ cells presented high levels of CD25/CD69 expression when the CD34+ cell lines KG1a and Kasumi-1 were co-cultured with $\mathrm{T}$ cells in the presence of CD34/CD3 BTE (Figure 3A). No unspecific activation was found when the CD34- NALM6 cell line was used as target cells. In the same way, T cells only presented significant expansion when cultured with CD34-expressing target cells with CD34/CD3 BTE (Figure 3B, C), indicating that the T-cell activation is CD34-dependent.

\section{The CD34-bi-specific T-cell engager depletes hematopoietic stem cells}

Since CD34 is constitutively expressed by HSC, the CD34specific BTE may deplete not only CD34 ${ }^{+} A M L$ blasts but also healthy HSC. To test this, effector T cells and HSC were purified from the same peripheral blood stem cell grafts and co-cultured in the presence of either the aCD34 antibody, RSV/CD3 BTE or the CD34/CD3 BTE. After co-culture, a significant depletion of CD34+ HSC was observed for the CD34/CD3 BTE, while no significant killing was observed in the presence of the $\alpha$ CD34 antibody or the RSV/CD3 BTE (Figure 4A, B). This was accompanied by a dose-dependent increase of activated $C D 4^{+} C D 25^{+} \mathrm{CD} 69^{+}$ and $\mathrm{CD}^{+} \mathrm{CD} 25^{+} \mathrm{CD} 69^{+} \mathrm{T}$ cells in the CD34-BTE-treated conditions, while no activation was observed in the controls (Figure 4C, D). These data indicate that the CD3/CD34 BTE induces T-cell-mediated HSC killing.

\section{The CD34-bi-specific T-cell engager depletes human CD $34^{+}$blast cells from acute myeloid leukemia patients ex vivo}

CD $34^{+}$blast count in the peripheral blood and bone marrow is used for diagnosis and prognosis of AML. ${ }^{1}$ In order to test the ability of the CD34-specific BTE to deplete CD34+ blasts, we co-cultured effector $\mathrm{T}$ cells isolated from donor lymphocyte infusions with target CD34+ $\mathrm{AML}$ blasts from primary patients' samples. We initially compared an incubation time of $48 \mathrm{~h}$ versus $72 \mathrm{~h}$ in the presence of several concentrations of CD34 BTE and corresponding controls, with the later timepoint presenting clearer results regarding $T$-cell activation and blast killing, being adopted thereafter (Online Supplementary Figure S4). We observed no CD34+ blast depletion in either the $\alpha$ CD34- or RSV/CD3-treated group (Figure 5A), despite the increase of activated T cells in the RSV/CD3 control group (Figure 5B). The CD34/CD3 BTE triggered both significant T-cell activation and killing of CD34+ blasts (Figure $5 \mathrm{~A}, \mathrm{~B}$ ), resulting in significant blast reduction (Figure $5 \mathrm{C}$ ). No correlation was observed between blast reduction and CD34 expression (Figure 5D). Dose-dependent T-cell activation and blast depletion were observed when $T$ cells from five different donors were cultured with bone marrow-derived CD34+ blasts from AML patients (Online Supplementary Figure S5), together suggesting that the BTE can trigger efficient T-cell-mediated depletion of primary CD34+ AML blasts ex vivo.

Next, we used the cell line hCMEC/D3 as the target to test whether the developed CD34 BTE can promote the killing 

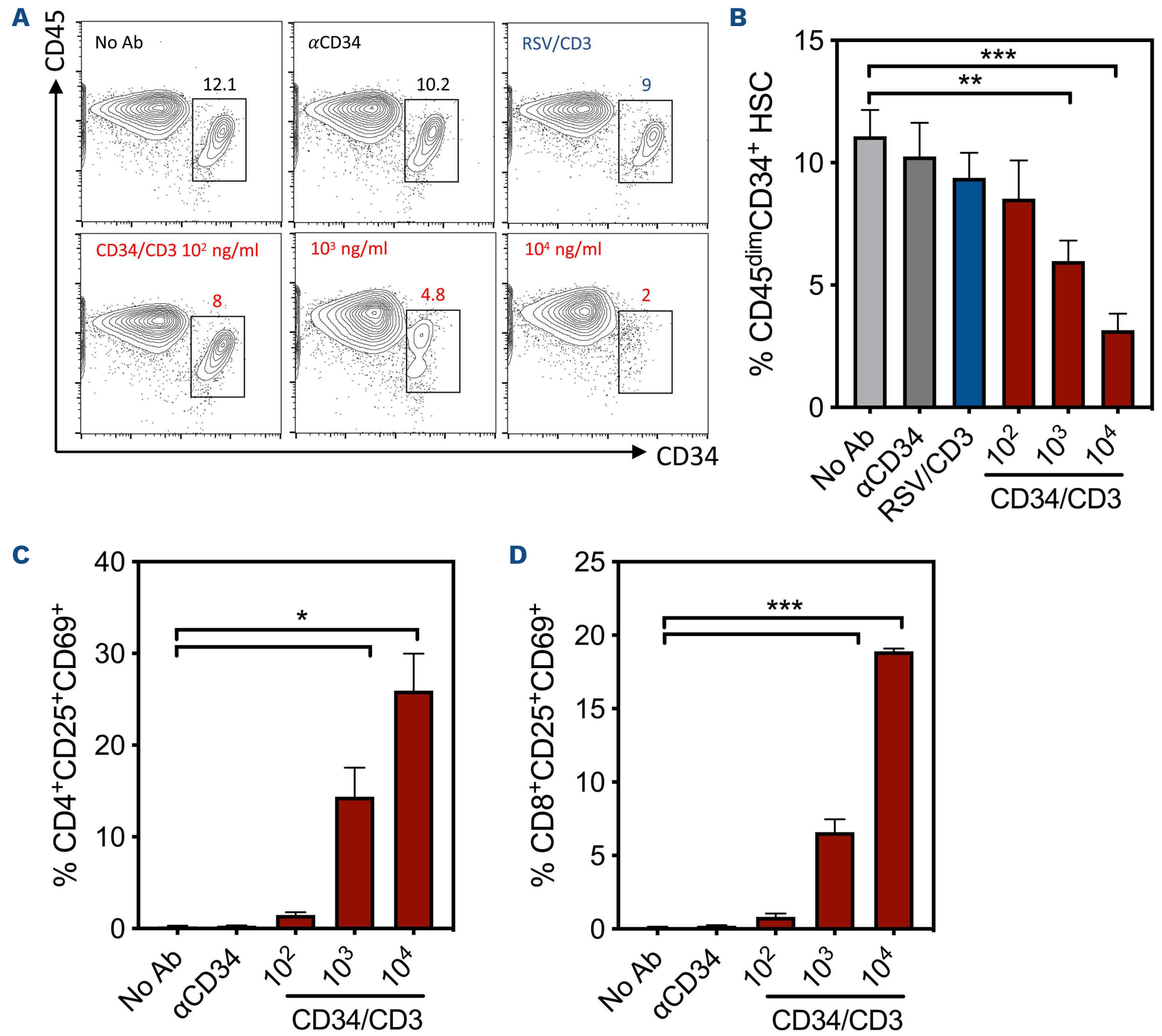

Figure 4. The CD34-specific bi-specific T-cell engager efficiently depletes hematopoietic stem cells. T cells and CD34 ${ }^{+}$hematopoietic stem cells (HSC) were isolated from the same peripheral blood stem cell grafts and co-cultured at an effector:target ratio of 3:1 in the presence of anti-CD34 antibody, RSV/CD3, CD34/CD3 from $10^{2}$ to $10^{4} \mathrm{ng} / \mathrm{mL}$ or left untreated. (A) Representative plot of $\mathrm{CD} 45^{\mathrm{dim}} \mathrm{CD} 34^{+} \mathrm{HSC}$ quantification after $48 \mathrm{~h}$ of co-culture. (B) Quantification of CD45 ${ }^{\mathrm{dim}} \mathrm{CD} 34^{+} \mathrm{HSC}$ depletion (mean \pm standard error of mean [SEM], $n=6$ ). (C) $C D 3^{+} C D 4^{+} C D 25^{+} C D 69^{+}$and (D) $C D 3^{+} C D 8^{+} C D 25^{+} C D 69^{+}$activated $T$ cells separated by fluorescence activated cell sorting (mean $\pm \mathrm{SEM}, \mathrm{n}=6$ ). $* P<0.05, * * P<0.01, * \star * P<0.001$ (Wilcoxon test).

of human $\mathrm{CD}_{3} 4^{+}$endothelial cells. We found that, even at high concentrations $\left(10^{3} \mathrm{ng} / \mathrm{mL}\right)$, the CD34 BTE was not able to deplete the endothelial cells (Online Supplementary Figure S6), indicating that the BTE does not crossreact with $\mathrm{CD} 34$ protein expressed by endothelial cells.

\section{In vivo efficacy of the bi-specific T-cell engager}

To address the potential of the anti-CD34 BTE in vivo, we next established the hCD34+ ${ }^{+}$KG1a cell line in NSG-SGM3 mice via intravenous injection and then randomized them into three different groups. Two groups of mice received two consecutive cycles of one intraperitoneal injection of freshly isolated human $T$ cells followed by daily intravenous injections of either BTE or phosphate-buffered saline (PBS) (Figure 6A). One group only received daily intravenous injections of PBS, at the time point corresponding to that of BTE, but no $T$ cells. The mice were euthanized at the predetermined time on day 21, at which point the AML burden was measured, and the T cells quantified. No side effects of the treatment, including after BTE administration, were observed.

At day 21 there were statistically significant reductions of leukemia burden in both bone marrow (Figure 6B) and spleen (Figure $6 \mathrm{C}$ ) in mice receiving $T$ cells and BTE compared to those that received T cells only and PBS control. Of note, the leukemia clearance was near complete in the bone marrow of mice receiving the combination of $T$ cells and BTE (Figure 6B). As we had observed tumor reduction in both these groups compared to the tumor burden in the group given PBS only, we hypothesized that this was primarily attributed to the presence of $T$ cells. To address this, we next analyzed the T-cell compartment in the harvested organs by flow cytometry. The data revealed persistence of $T$ cells in mice treated with the CD34/CD3 BTE, 

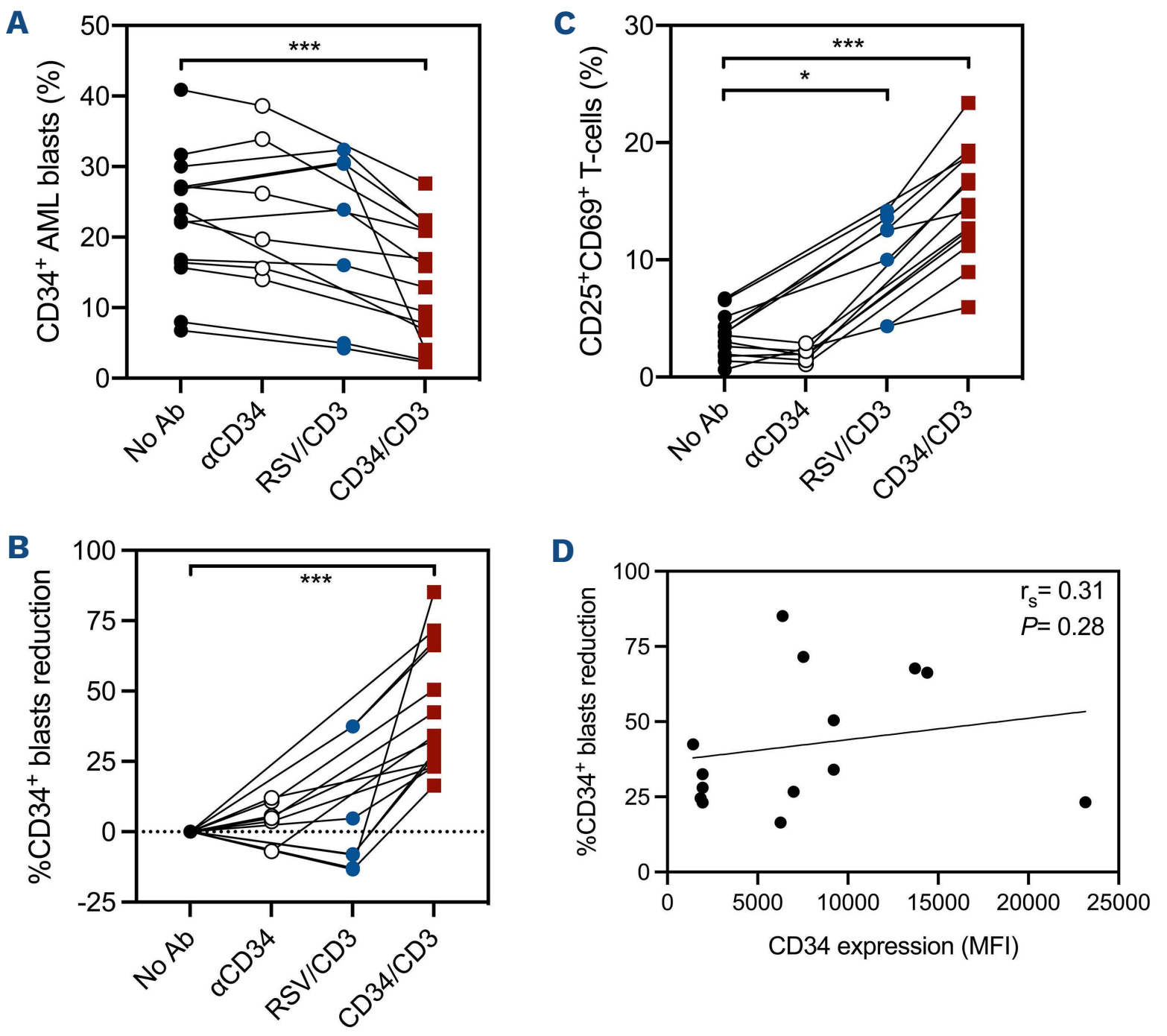

Figure 5. Bi-specific T-cell engager treatment leads to depletion of patients' CD $34^{+}$acute myeloid leukemia blasts. (A) Purified T cells from donor lymphocyte infusions were co-cultured with $\mathrm{CD} 34^{+}$blasts isolated from patients with acute myeloid leukemia in the presence of anti-CD34 antibody, RSV/CD3 or CD34/CD3 bi-specific T-cell engagers (BTE) at a concentration of $2.5 \mu \mathrm{g} / \mathrm{mL}$ for $72 \mathrm{~h}(\mathrm{n}=14)$. (B) Quantification of $C D 3^{+} C D 25^{+} C D 69^{+}$activated T cells $(n=14)$. (C) Percentage CD34 ${ }^{+}$blast reduction as compared to untreated (No Ab) control. (D) Correlation between blast reduction and CD34 expression (Spearman rank correlation coefficient). Dots represent individual patients and the connecting lines the paired samples. ${ }^{*} P<0.05, * \star \star P<0.001$ (Wilcoxon test).

while the PBS-treated mice had a very low T-cell frequency $(<1 \%$ in all mice) but, in contrast, high counts of CD34+ blasts (Figure 6C, D).

Collectively, these data show that $\mathrm{hCD} 34^{+} \mathrm{AML}$-engrafted mice treated with the combination of primary human $T$ cells and the CD3/CD34 BTE have significantly reduced tumor burden associated with T-cell persistence, without any side effects in the mice.

\section{Discussion}

Leukemia-specific BTE have been developed intensively in the last years with prominent in vitro and in vivo effects by targeting $\mathrm{CD} 33,{ }^{25} \mathrm{CD} 123^{26}$ and $\mathrm{WT} 1^{27}$ in AML and CD19 in B-cell acute lymphoblastic leukemia. ${ }^{21}$ We have developed a CD34-specific BTE aiming to deplete both CD34-expressing HSC and leukemic blasts, including LSC. Here we have shown that the CD34/CD3 BTE is able to promote Tcell activation and killing of CD34-expressing target cells with high efficacy in vitro and in vivo, supporting the translation of this drug into clinical trials.
In patients with high-risk MDS/AML, HSCT is the only curative treatment. Disease relapse, infections and graftversus-host disease are still the major causes of treatment failure, with few improvements in the last years. ${ }^{35}$ This indicates that adjuvant therapies are necessary to make HSCT safer and new approaches are required to treat relapsed patients after HSCT. CD $34^{+}$leukemic cell frequency has been associated with high relapse rates, ${ }^{10-13}$ implying that the use of leukemia-depleting drugs in HSCT protocols could be beneficial to reduce the tumor burden. In this scenario, treatment with CD34-targeting BTE prior to HSCT would trigger the patient's T cells to deplete CD34 ${ }^{+}$leukemic blasts, LSC and HSC. As a consequence, this adjuvant treatment would decrease the use of cytotoxic and cytostatic conditioning drugs before HSCT, reducing life-threatening complications such as infections. Recently, an anti-CD117 monoclonal antibody has been used with non-myeloablative conditioning in patients with minimal residual disease-positive MDS/AML undergoing allogeneic HSCT. ${ }^{36}$ The treatment has been shown to be safe, well-tolerated and efficient in clearing MDS/AML minimal residual disease, promoting complete donor 
A

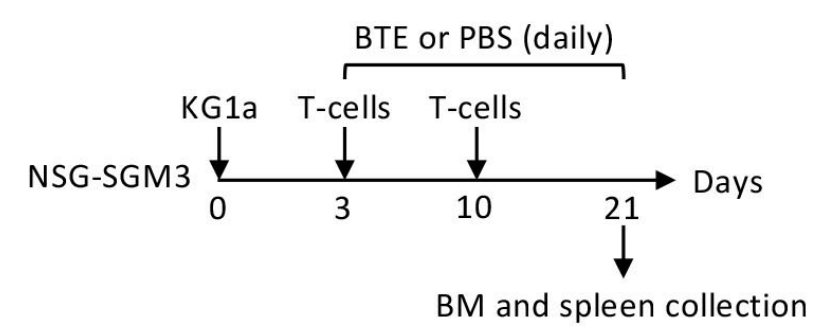

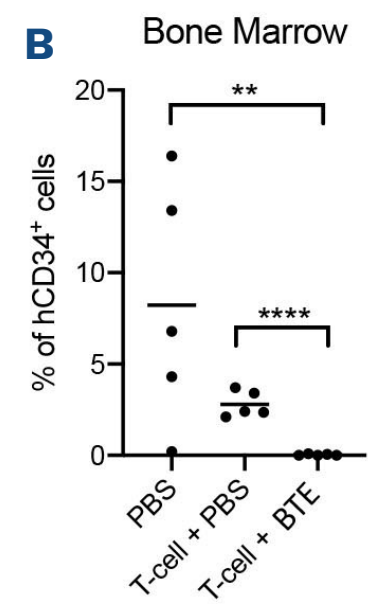
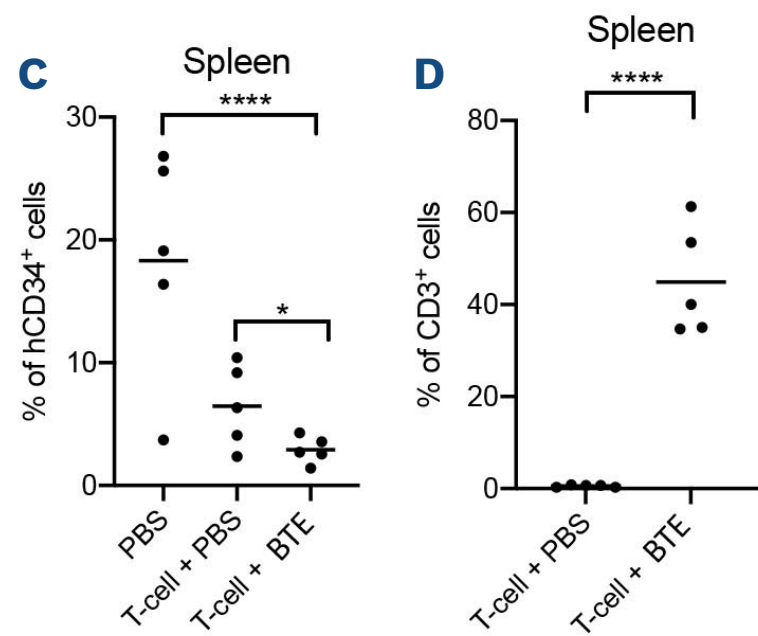

Figure 6. In vivo efficacy of the bi-specific T-cell engager against acute myeloid leukemia in a xenograft mouse model. (A) Schematic overview of the protocol, in which NSG-SGM3 mice were inoculated with the hCD34+ acute myeloid leukemia (AML) cell line KG1a intravenously before receiving two cycles of freshly isolated human T cells intraperitoneally (days 3 and 10) and either $1 \mathrm{mg} / \mathrm{kg}$ of bi-specific T-cell engager (BTE) or phosphate-buffered saline (PBS) daily intravenously (day 3 to 21). AML burden and T-cell persistence were assessed by organ harvest and flow cytometry at the predetermined termination day 21 . Quantification of hCD34+ leukemia cells in (B) bone marrow and (C) spleen. (D) Quantification on hCD3 ${ }^{+} \mathrm{T}$ cells in spleen. Five mice per group were analyzed. $* * P<0.01, * * * P<0.001, * * * * P<0.0001$ (unpaired $t$-test).

chimerism. ${ }^{36}$ Less than $25 \%$ of patients who could benefit from HSCT actually undergo transplantation because of the high toxicity associated with conditioning regimens. ${ }^{37}$ There are indications that adjuvant antibody-based therapies, such as the here presented CD34 BTE, can reduce conditioning toxicities and expand the use of HSCT to older and more fragile patients.

CD34 is expressed in other tissues besides HSC and blasts, and a potential side effect of targeting CD34 is the recognition of $\mathrm{CD}_{3} 4^{+}$endothelial cells. ${ }^{38}$ Considering that BTE have a relatively short half-life $(\approx 2 h)$, possible side effects could be circumvented by halting drug administration. Moreover, we show here that the BTE developed did not present significant activity against a human $\mathrm{CD} 34^{+}$endothelial cell line even at high concentrations, indicating that our CD34-specific BTE is specific for CD34 expressed by cells from the hematologic lineage rather than from the endothelial lineage. More studies, including patientderived xenograft models, humanized mice models $\left(\mathrm{hCD} 34^{+}\right)$and non-human primates are necessary to confirm these observations. This lack of reactivity could be explained by the presence of several CD34 epitopes that are differentially expressed in specific tissues as results of heavy glycosylation, ${ }^{39,40}$ and therefore can be targeted by specific antibodies. ${ }^{41}$ Additionally, further engineering of the CD34-specific SCFV might improve BTE affinity to CD34 expressed by leukemic blasts and HSC rather than endothelial CD34.

The CD34-specific BTE developed could also be used in the treatment of a wide range of non-malignant diseases as an HSC-specific depleting agent in a non-myeloablative conditioning treatment regimen, as shown by the use of anti-CD117 antibodies..$^{42,43}$ This would be of particular interest in cases of inborn mutations that can be cured by gene therapy or HSCT in which reduced intensity chemotherapy is desired. In this case, the CD34-specific BTE could be used as adjuvant therapy prior to HSC, and combined with anti-T-cell antibodies, it may be possible to significantly reduce chemotherapy intensity during conditioning therapy. This would result in significantly reduced short- and long-term side effects of chemotherapy following HSCT. In this regard, there are recent reports that the use of anti-CD117 as non-toxic single-agent transplant conditioning may supplant conventional conditioning for newly diagnosed infants with severe combined immunodeficiency, thereby avoiding the toxicities of chemotherapy. ${ }^{44,45}$

Currently, HSCT is still restricted to patients with otherwise incurable malignant diseases and it is not indicated for most of the patients who could benefit from it because of its high risks and toxicity. ${ }^{37}$ This is largely due to the use of non-specific cytotoxic chemotherapy and the irradiation conditioning necessary to enable engraftment of donor HSC. Regular conditioning regimens lead to longterm immune deficiency and multi-organ damage, which is associated with a high risk of infections. ${ }^{46}$ This is particularly severe in children, in whom conditioning regimens are associated with infertility, hormonal dysfunction and growth problems. ${ }^{47}$ The developed CD34specific BTE could help to eliminate such severe conditioning regimens and dramatically improve HSCT and expand its use.

\section{Disclosure}

JED receives royalties from Trillium Therapeutics Inc and reports receiving a commercial research grant from Celgene. The other authors declare that they have no competing financial interests. 


\section{Contributions}

LCMA, AS and RAR performed in vitro experiments with cell lines and human hematopoietic stem cells. AS and LJ conducted the in vitro studies with primary acute myeloid leukemia samples. ML performed the in vivo experiments. $L S R$ and $R A$ performed the single-cell killing experiments. TRM and CM purified the antibodies. SL, JED, JM, BO, MC, and MU provided financial support. LCMA and MU designed the antibodies and wrote the manuscript. All authors critically reviewed the manuscript and approved the final ver- sion. AS, ML and LJ contributed equally to this work.

\section{Funding}

This work was supported by research funding from Stiftelsen Felix Mindus Bidrag till Leukemiforskningen, David och Astrid Hageléns Stiftelse, Wallenberg, Cancerfonden, and Barncancerfonden. Vetenskapsrådet (2021-01755)

\section{Data-sharing statement}

Data are available upon request to the author.

\section{References}

1. Short NJ, Rytting ME, Cortes JE. Acute myeloid leukaemia. Lancet. 2018;392(10147):593-606.

2. Doll DC, List AF. Myelodysplastic syndromes. West J Med. 1989;151(2):161-167.

3. Cancer Stat Facts: leukemia - acute myeloid leukemia (AML). https://seer.cancer.gov/statfacts/html/amyl.html.

4. Vetrie D, Helgason GV, Copland M. The leukaemia stem cell: similarities, differences and clinical prospects in $\mathrm{CML}$ and $\mathrm{AML}$. Nat Rev Cancer. 2020;20(3):158-173.

5. Lapidot T, Sirard C, Vormoor J, et al. A cell initiating human acute myeloid leukaemia after transplantation into SCID mice. Nature. 1994;367(6464):645-648.

6. Bonnet D, Dick JE. Human acute myeloid leukemia is organized as a hierarchy that originates from a primitive hematopoietic cell. Nat Med. 1997;3(7):730-737.

7. Shlush LI, Mitchell A, Heisler L, et al. Tracing the origins of relapse in acute myeloid leukaemia to stem cells. Nature. 2017;547(7661):104-108.

8. Ho TC, LaMere M, Stevens BM, et al. Evolution of acute myelogenous leukemia stem cell properties after treatment and progression. Blood. 2016;128(13):1671-1678.

9. Ng SWK, Mitchell A, Kennedy JA, et al. A 17-gene stemness score for rapid determination of risk in acute leukaemia. Nature. 2016;540(7633):433-437.

10. Van Rhenen A, Feller N, Kelder A, et al. High stem cell frequency in acute myeloid leukemia at diagnosis predicts high minimal residual disease and poor survival. Clin Cancer Res. 2005;11(18):6520-6527.

11. Jentzsch M, Geus U, Grimm J, et al. Pretreatment CD34+/CD38- cell burden as prognostic factor in myelodysplastic syndrome patients receiving allogeneic stem cell transplantation. Biol Blood Marrow Transplant. 2019;25(8):1560-1566.

12. Jentzsch M, Bill M, Nicolet $D$, et al. Prognostic impact of the CD34+/CD38- cell burden in patients with acute myeloid leukemia receiving allogeneic stem cell transplantation. Am J Hematol. 2017;92(4):388-396.

13. Zeijlemaker W, Grob T, Meijer R, et al. CD34+CD38- leukemic stem cell frequency to predict outcome in acute myeloid leukemia. Leukemia. 2019;33(5):1102-1112.

14. Saygin C, Matei D, Majeti R, Reizes O, Lathia JD. Targeting cancer stemness in the clinic: rrom hype to hope. Cell Stem Cell. 2019;24(1):25-40.

15. Velasquez MP, Bonifant CL, Gottschalk S. Redirecting T cells to hematological malignancies with bi-specific antibodies. Blood. 2018;131(1):30-38.

16. Labrijn AF, Janmaat ML, Reichert JM, Parren PWHI. Bispecific antibodies: a mechanistic review of the pipeline. Nat Rev Drug Discov. 2019;18(8):585-608.

17. Einsele H, Borghaei H, Orlowski RZ, et al. The BTE (bi-specific T-cell engager) platform: development and future potential of a targeted immuno-oncology therapy across tumor types. Cancer. 2020;126(14):3192-3201.

18. Costello RT, Mallet F, Gaugler B, et al. Human acute myeloid leukemia CD34+/CD38- progenitor cells have decreased sensitivity to chemotherapy and Fas-induced apoptosis, reduced immunogenicity, and impaired dendritic cell transformation capacities. Cancer Res. 2000;60(16):4403-4411.

19. She M, Niu X, Chen X, et al. Resistance of leukemic stem-like cells in AML cell line KG1a to natural killer cell-mediated cytotoxicity. Cancer Lett. 2012;318(2):173-179.

20. Raaijmakers MHGP, De Grouw EPLM, Heuver LHH, et al. Breast cancer resistance protein in drug resistance of primitive CD34+38- cells in acute myeloid leukemia. Clin Cancer Res. 2005;11(6):2436-2444.

21. Gökbuget N, Dombret $H$, Bonifacio $M$, et al. Blinatumomab for minimal residual disease in adults with $\mathrm{B}$-cell precursor acute lymphoblastic leukemia. Blood. 2018;131(14):1522-1531.

22. Bargou R, Leo E, Zugmaier G, et al. Tumor regression in cancer patients by very low doses of a T cell-engaging antibody. Science. 2008;321(5891):974-977.

23. Löffler $A$, Kufer $P$, Lutterbüse $R$, et al. A recombinant bi-specific single-chain antibody, CD19 $\times$ CD3, induces rapid and high lymphoma-directed cytotoxicity by unstimulated $T$ lymphocytes. Blood. 2000;95(6):2098-2103.

24. Norsworthy KJ, Ko C-W, Lee JE, et al. FDA approval summary: mylotarg for treatment of patients with relapsed or refractory CD33-positive acute myeloid leukemia. Oncologist. 2018;23(9):1103-1108.

25. Aigner M, Feulner J, Schaffer S, et al. T lymphocytes can be effectively recruited for ex vivo and in vivo lysis of AML blasts by a novel CD33/CD3-bi-specific BTE antibody construct. Leukemia. 2013;27(5):1107-1115.

26. Hutmacher C, Volta L, Rinaldi F, et al. Development of a novel fully-human anti-CD123 antibody to target acute myeloid leukemia. Leuk Res. 2019;84:106178.

27. Dao T, Pankov D, Scott A, et al. Therapeutic bi-specific T-cell engager antibody targeting the intracellular oncoprotein WT1. Nat Biotechnol. 2015;33(10):1079-1086.

28. Zeijlemaker W, Kelder A, Wouters R, et al. Absence of leukaemic CD34+ cells in acute myeloid leukaemia is of high prognostic value: a longstanding controversy deciphered. $\mathrm{Br} \mathrm{J}$ Haematol. 2015;171(2):227-238.

29. Suárez L, Vidriales MB, García-Laraña J, et al. CD34+ cells from 
acute myeloid leukemia, myelodysplastic syndromes, and normal bone marrow display different apoptosis and drug resistance - associated phenotypes. Clin Cancer Res. 2004;10(22):7599-7606.

30. Robinson HR, Qi J, Cook EM, et al. A CD19/CD3 bi-specific antibody for effective immunotherapy of chronic lymphocytic leukemia in the ibrutinib era. Blood. 2018;132(5):521-532.

31. Hipp S, Tai Y-T, Blanset D, et al. A novel BCMA/CD3 bi-specific Tcell engager for the treatment of multiple myeloma induces selective lysis in vitro and in vivo. Leukemia. 2017;31(8):1743-1751.

32. Sarhan D, Brandt L, Felices M, et al. 161533 TriKE stimulates NKcell function to overcome myeloid-derived suppressor cells in MDS. Blood Adv. 2018;2(12):1459-1469.

33. Guldevall K, Brandt L, Forslund E, et al. Microchip screening platform for single cell assessment of NK cell cytotoxicity. Front Immunol. 2016;7(119):1-7.

34. Arruda LCM, Gaballa A, Uhlin M. Graft $\gamma \delta$ TCR sequencing identifies public clonotypes associated with hematopoietic stem cell transplantation efficacy in acute myeloid leukemia patients and unravels cytomegalovirus impact on repertoire distribution. J Immunol. 2019;202(6):1859-1870.

35. Horowitz M, Schreiber H, Elder A, et al. Epidemiology and biology of relapse after stem cell transplantation. Bone Marrow Transplant. 2018;53(11):1379-1389.

36. Muffly LS, Chin M, Kwon H-S, et al. Early results of phase 1 study of JSP191, an anti-CD117 monoclonal antibody, with nonmyeloablative conditioning in older adults with MRD-positive MDS/AML undergoing allogeneic hematopoietic cell transplantation. J Clin Oncol. 2021;39(15_suppl):7035.

37. Yao S, Hahn T, Zhang Y, et al. Unrelated donor allogeneic hematopoietic cell transplantation is underused as a curative therapy in eligible patients from the United States. Biol Blood Marrow Transplant. 2013;19(10):1459-1464.

38. Uhlén M, Fagerberg L, Hallström BM, et al. Proteomics. tissuebased map of the human proteome. Science.
2015;347(6220):1260419.

39. Croockewit AJ, Raymakers RA, Preijers FW, Vierwinden G, De Witte TJ. The role of the different CD34 epitopes in detection and positive selection of CD34 + bone marrow and peripheral blood stem cells. Scand J Immunol. 1998;47(1):82-90.

40. Steen R, Tjønnfjord GE, Gaudernack G, Brinch L, Egeland T. Differences in the distribution of CD34 epitopes on normal haemopoietic progenitor cells and leukaemic blast cells. $\mathrm{Br} J$ Haematol. 1996;94(4):597-605.

41. Sidney LE, Branch MJ, Dunphy SE, Dua HS, Hopkinson A. Concise review: evidence for CD34 as a common marker for diverse progenitors. Stem Cells. 2014;32(6):1380-1389.

42. Chhabra A, Ring AM, Weiskopf K, et al. Hematopoietic stem cell transplantation in immunocompetent hosts without radiation or chemotherapy. Sci Transl Med. 2016;8(351):351ra105.

43. Czechowicz A, Palchaudhuri R, Scheck A, et al. Selective hematopoietic stem cell ablation using CD117-antibody-drugconjugates enables safe and effective transplantation with immunity preservation. Nat Commun. 2019;10(1):617.

44. Agarwal R, Weinberg KI, Kwon H-S, et al. First report of nongenotoxic conditioning with JSP191 (anti-CD117) and hematopoietic stem cell transplantation in a newly diagnosed patient with severe combined immune deficiency. Blood. 2020;136(Suppl 1):10.

45. Agarwal R, Dvorak CC, Kwon H-S, et al. Non-genotoxic antiCD117 antibody conditioning results in successful hematopoietic stem cell engraftment in patients with severe combined immunodeficiency. Blood. 2019;134(Suppl_1):800.

46. Bearman SI, Appelbaum FR, Buckner CD, et al. Regimen-related toxicity in patients undergoing bone marrow transplantation. $J$ Clin Oncol. 1988;6(10):1562-1568.

47. Matthes-Martin S, Lamche M, Ladenstein R, et al. Organ toxicity and quality of life after allogeneic bone marrow transplantation in pediatric patients: a single centre retrospective analysis. Bone Marrow Transplant. 1999;23(10):1049-1053. 